\title{
De novo assembly and characterization of transcriptome using Illumina paired-end sequencing and identification of CesA gene in ramie (Boehmeria nivea L. Gaud)
}

\author{
Touming Liu, Siyuan Zhu, Qingming Tang, Ping Chen, Yongting Yu and Shouwei Tang*
}

\begin{abstract}
Background: Ramie fiber, extracted from vegetative organ stem bast, is one of the most important natural fibers. Understanding the molecular mechanisms of the vegetative growth of the ramie and the formation and development of bast fiber is essential for improving the yield and quality of the ramie fiber. However, only 418 expressed tag sequences (ESTs) of ramie deposited in public databases are far from sufficient to understand the molecular mechanisms. Thus, high-throughput transcriptome sequencing is essential to generate enormous ramie transcript sequences for the purpose of gene discovery, especially genes such as the cellulose synthase (CesA) gene.
\end{abstract}

Results: Using Illumina paired-end sequencing, about 53 million sequencing reads were generated. De novo assembly yielded 43,990 unigenes with an average length of 824 bp. By sequence similarity searching for known proteins, a total of 34,192 (77.7\%) genes were annotated for their function. Out of these annotated unigenes, 16,050 and 13,042 unigenes were assigned to gene ontology and clusters of orthologous group, respectively. Searching against the Kyoto Encyclopedia of Genes and Genomes Pathway database (KEGG) indicated that 19,846 unigenes were mapped to $126 \mathrm{KEGG}$ pathways, and 565 genes were assigned to starch and sucrose metabolic pathway which was related with cellulose biosynthesis. Additionally, 51 CesA genes involved in cellulose biosynthesis were identified. Analysis of tissue-specific expression pattern of the 51 CesA genes revealed that there were 36 genes with a relatively high expression levels in the stem bark, which suggests that they are most likely responsible for the biosynthesis of bast fiber.

Conclusion: To the best of our knowledge, this study is the first to characterize the ramie transcriptome and the substantial amount of transcripts obtained will accelerate the understanding of the ramie vegetative growth and development mechanism. Moreover, discovery of the 36 CesA genes with relatively high expression levels in the stem bark will present an opportunity to understand the ramie bast fiber formation and development mechanisms.

\section{Background}

Sequencing and analysis of expressed sequence tags (ESTs) has been a primary tool in gene discovery and genomic sequence annotation in plants. Additionally, ESTs can be used for other functional genomic projects, including gene expression profiling, microarrays, molecular markers, and physical mapping. Over the past ten years, in order to obtain the transcribe EST information, a large number of

\footnotetext{
* Correspondence: cesc2012@yahoo.cn

Institute of Bast Fiber Crops and Center of Southern Economic Crops,

Chinese Academy of Agricultural Sciences, Changsha 410205, China
}

cDNA libraries have been constructed and sequenced for rice [1], maize [2,3], wheat [4] and other crops. However, traditional sequencing methods used for the generation of ESTs require costly and time-consuming approaches involving cDNA library construction, cloning, and the labor-intensive Sanger sequencing. Alternatively, the transcriptome analysis based on the next generation sequencing (NGS) is more attractive in identifying the expression gene for its characteristics of cost-efficient, high throughput and rapidness. NGS, including the Roche/454 Genome 
Sequencer FLX Instrument, the ABI SOLiD System and the Illumina Genome Analyser, was a powerful tool and was untilized in many researching areas, including resequencing, micro-RNA expression profiling, DNA methylation, especially de novo transcriptome sequencing for non-model organisms [5-13]. Recently, based on the NGS technology, the transcriptome of many species such as chestnut [9], coral larval [5], sweetpotato [11], ginseng root [10], chickpea [12] and saccharina japonica [13] had been analyzed, which has accelerated our understanding of the complexity of expression, regulation and networks of gene in model and non-model organisms.

Ramie (Boehmeria nivea), popularly named as "Chinagrass", is a perennial diploid $(2 \mathrm{n}=28)$ herbaceous plant belonging to the family of Urticaceae and is an important natural fiber crop. Ramie fibers extracted from stem bast possess characteristics such as smooth texture, long strands and excellent tensile strength, which makes ramie to be widely planted in China, India, and other Southeast Asian and Pacific Rim countries. In China, ramie is the second major fiber crop and its growth acreage and fiber production are surpassed only by those of cotton. Ramie has a vigorous vegetative growth and can be harvested three times per year in China, and up to six times per year in well-watered cultivation environments in Philippines, which allow ramie to produce a high yield of vegetative fiber. Therefore, understanding the processes regulating vegetative growth and development of ramie is valuable. Moreover, the ramie bast fiber, involved in sugar metabolism and cellulose synthesis, is an important organ, which has the value of biological research for organogenesis and evolution. During the last decade, a large number of transcriptomic and genomic sequences became available in model organisms, such as Arabidopsis and rice, which has greatly improved the understanding of the complexity of growth and development in higher plants. However, for ramie, only 418 EST sequences have been deposited in the GenBank database (as of August 2012). Obviously, the public available data is far from sufficient to understand the molecular mechanisms involved in vegetative development and fiber biosynthesis in ramie. Therefore, extensive transcriptomic sequence data are essential for ramie, which can be used to discover a large number of new genes.

In the present study, we utilized the Illumina paired-end sequencing technology to characterize the transcriptome of ramie and identify the cellulose synthase (CesA) gene. Non-normalized cDNA collections from different types of tissues were used to generate a broad survey of genes associated with ramie vegetative growth and development. To the best of our knowledge, this study is the first exploration to characterize the transcriptome of ramie through the analysis of large-scale transcript sequences resulting from Illumina paired-end sequencing.

\section{Result}

Illumina paired-end sequencing and de novo assembly

In order to characterize the transcriptome of ramie and generate a broad survey of genes associated with ramie vegetative growth and development, total RNA was extracted from several vegetative tissues including leaf, root, stem bast, stem xylem and stem shoot at vegetative stages. Using Illumina paired-end sequencing technology, each sequencing feature can yield $2 \times 90$ bp independent reads from the either end of a DNA fragment. In this study, a total of 57,471,036 raw sequencing reads with the length of $90 \mathrm{bp}$ were generated from a $200 \mathrm{bp}$ insert library.

An assembler, Trinity developed specifically for use with next-generation short-read sequences [14], was employed for de novo assembly. After stringent quality checking and data cleaning, approximately 53 million high quality reads were obtained with $97.46 \%$ Q20 bases (base quality more than 20). Based on the high quality reads, 84,876 contigs were assembled with an average length of $393 \mathrm{bp}$ ranging from 100 to $17,496 \mathrm{bp}$. Of these, $44.8 \%$ and $11.6 \%$ contigs had a length more than $200 \mathrm{bp}$ and $1000 \mathrm{bp}$, respectively (Figure 1).

To obtain the unigenes, the paired-end reads were realigned to contigs and these contigs in one transcript were assembled by the Trinity and gained the sequence not being extended on the either end were defined as unigenes. Then the TGICL [15] was used to get rid of redundant unigene and to further assemble all the unigenes to form a single set of non-redundant unigenes. Finally the de novo assembly yielded 43,990 unigenes with an average length of $824 \mathrm{bp}$ and a total length of $36.26 \mathrm{Mb}$. The length of assembled unigenes ranged from 200 to 17,496 bp. There were 11,349 unigenes $(25.80 \%)$ with length no more than $300 \mathrm{bp}, 9,966$ unigenes $(22.66 \%)$ with length varying from 301 to $500 \mathrm{bp}, 10,081$ unigenes $(22.92 \%)$ in the length range of 501 to $1000 \mathrm{bp}$, and 12,564 unigenes (28.57\%) with length more than $1000 \mathrm{bp}$ (Figure 1). To evaluate the quality of the assembled unigenes, all the usable sequencing reads were realigned to the unigenes. The sequencing depth ranged from 0.1 to 132,686 folds, with an average of 62.98 folds. About $91.0 \%$ of the unigenes were realigned by more than 10 reads and $45.4 \%$ were remapped by more than 100 reads (Figure 2).

Sequence orientations of all unigenes were pridicted via ESTScan or BLAST (Basic Local Alignment Search Tool) with an E-value threshold of $10^{-5}$ in the NCBI database of non-redundant protein $(\mathrm{Nr})$, along with the Swiss-Prot protein database, the Kyoto Encyclopedia of Genes and Genomes (KEGG) database and the Clusters of Orthologous Groups (COG) database. Finally, sequence orientation of 34251 (77.8\%) unigenes was predicted and 9739 (22.2\%) unigenes sequence orientation is still unknown. 

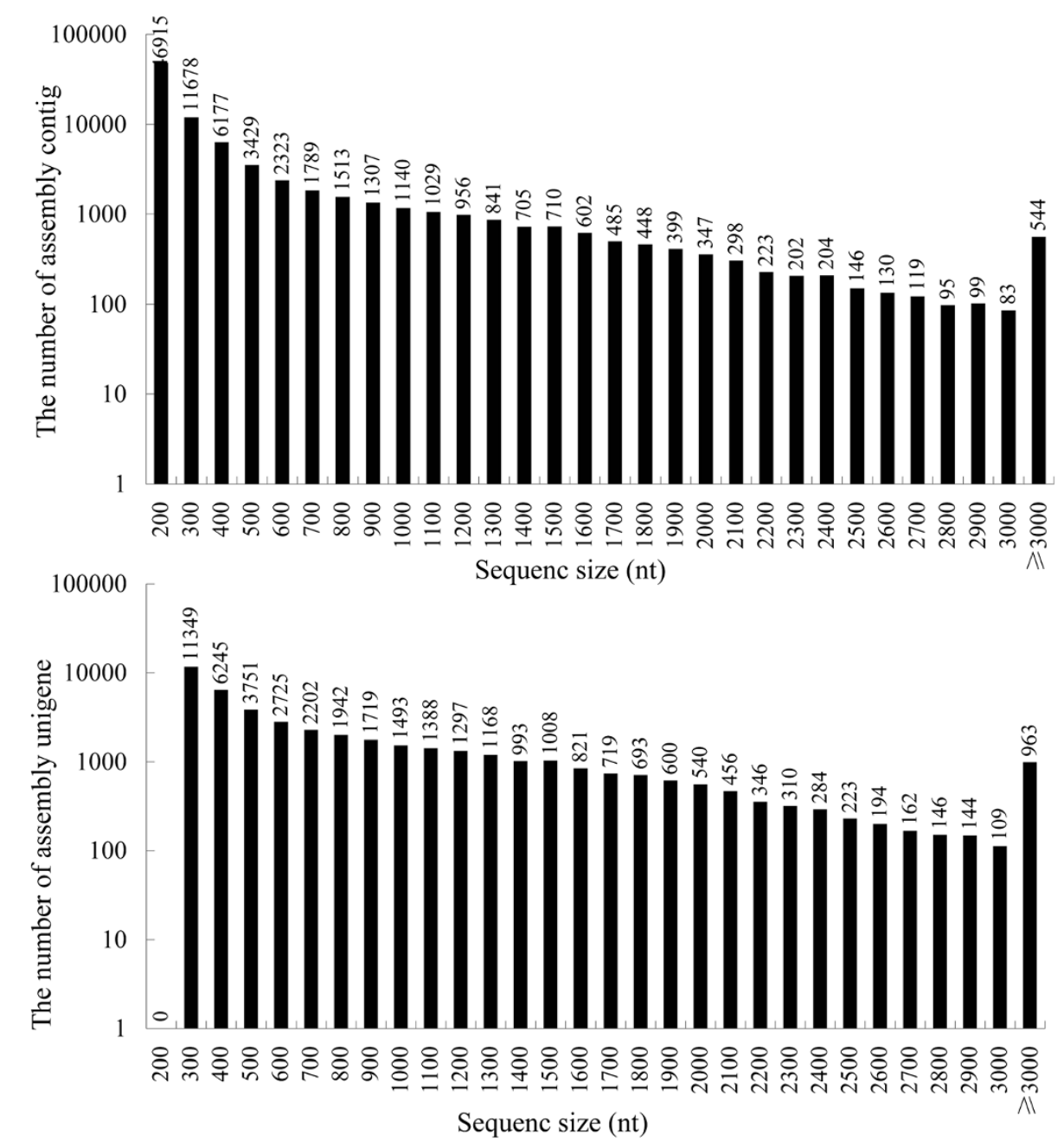

Figure 1 Length distribution of assembled contigs and unigenes.

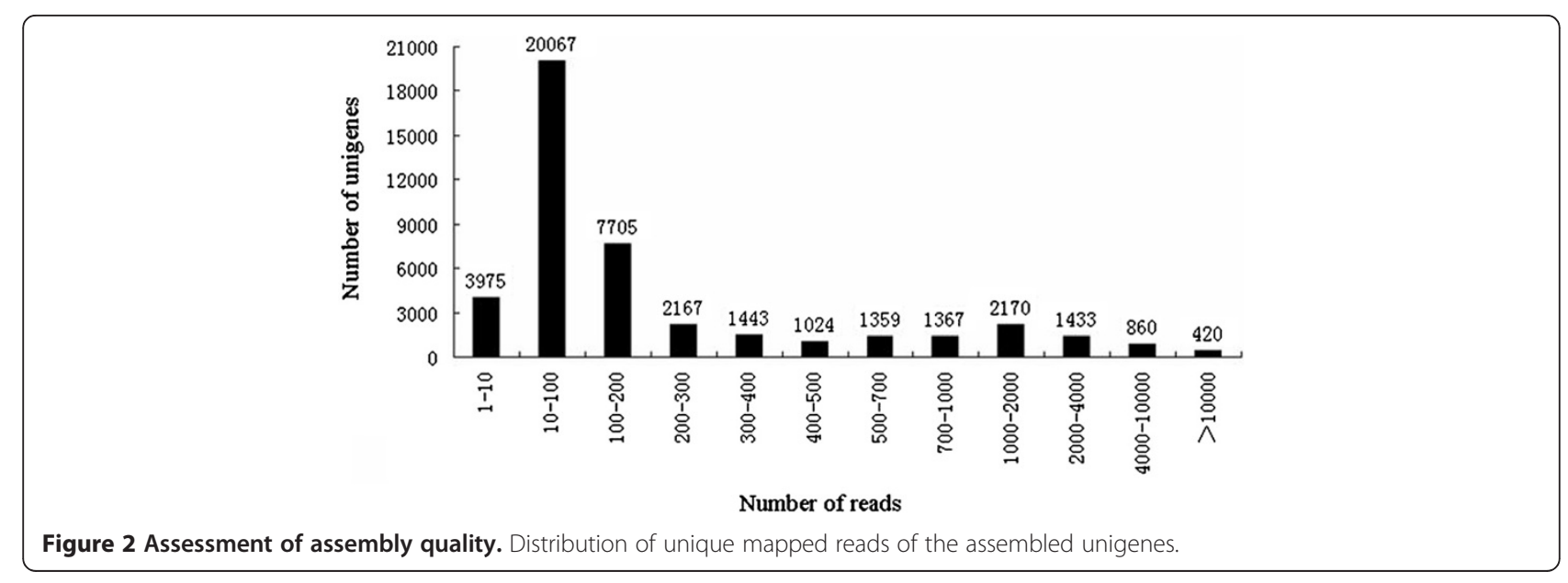


Functional annotation by searching against public databases For validation and annotation of the assembled unigenes, sequence similarity search was conducted in the Nr database, the COG database, the Swiss-Prot protein database, and the KEGG database $[16,17]$ with an E-value threshold of $10^{-5}$. The results indicated that out of 43,990 unigenes, 32,790 (74.5\%), 21,206 (48.2\%), 19,846 (45.1\%) and 13,042 (29.6\%) unigenes showed significant similarity to known proteins in Nr, SwissProt, KEGG and COG databases, respectively (Figure 3). Together, 34,192 (77.7\%) unigenes showed similarity to known proteins in four databases mentioned above. The E-value distribution of the top hits in the $\mathrm{Nr}$ database revealed that $57.39 \%$ of the mapped sequences showed significant homology (less than 1.0E-45) (Figure $4 \mathrm{~A}$ ), and $71.56 \%$ and $27.63 \%$ of the sequences with similarities greater than $60 \%$ and $80 \%$, respectively, were found (Figure 4B). Interestingly, 35.79\% of the unigenes showed significant homology with sequence of Vitis vinifera and $19.61 \%, 15.86 \%$ and $12.42 \%$ of the mapped sequences have a significant similarity with the sequence of Ricinus communis, Populus trichocarpa and Glycine max, respectively (Figure 4C).

\section{Functional classification by GO and COG}

Gene Ontology (GO) is an international standardized gene functional classification system which offers a dynamic-updated controlled vocabulary and a strictly defined concept to comprehensively describe the properties of genes and their products in any organism. GO has three ontologies: Molecular function, Cellular component and Biological process. On the basis of $\mathrm{Nr}$ annotation, the Blast2GO program [18] was used to obtain GO annotation for the unigenes annotated by the $\mathrm{Nr}$ database. Then the WEGO software [19] was used to perform GO functional classification for these unigenes. In total, 16,050 unigenes (36.5\%) with BLAST matches to known proteins were assigned to GO classes with 111,333 functional terms. Of these, assignments to the biological process made up the majority $(45,848,44.18 \%)$, followed by molecular function
(44,282, 39.77\%) and cellular component (21,203, 19.05\%, Figure 5).

The Clusters of Orthologous Groups (COG) is a database where the orthologous gene products were classified. Every protein in the COG database is assumed to be evolved from an ancestor protein, and the whole database is built on coding proteins with complete genome as well as system evolution relationships of bacteria, algae and eukaryotes. All unigenes were aligned to the COG database to predict and classify potential functions. Totally, 13,042 genes $(29.6 \%)$ were assigned to the 25 COG classifications (Figure 6). Some unigenes were assigned to several COG categories, which lead to a total of 30140 sequences assigned in 25 COG categories. Among the 25 COG categories, the cluster of General function prediction $(4,606,15.28 \%)$ represented the largest group, followed by Transcription $(2,999,9.95 \%)$, Replication, recombination and repair $(2,481,8.23 \%)$, Function unknown $(2,061$, $6.84 \%)$, Signal transduction mechanisms (2,038, 6.76\%), Translation, ribosomal structure and biogenesis $(2,023$, 6.71\%), Posttranslational modification, protein turnover, chaperones $(1,913,6.35 \%)$, Carbohydrate transport and metabolism $(1,810,6.01 \%)$ and Cell cycle control, cell division, chromosome partitioning (1,507, 5.00\%), Amino acid transport and metabolism (1,158, 3.84\%), whereas only a few unigenes were assigned to Nulcear structure and Extracellular structure (Figure 6).

\section{Metabolic pathway analysis by KEGG}

The Kyoto Encyclopedia of Genes and Genomes (KEGG) Pathway database records the networks of molecular interactions in the cells, and variants of them specific to particular organisms. Pathway-based analysis helps us to further understand the biological functions and interactions of genes. First, based on a comparison against the KEGG database using BLASTx with an E-value threshold of $10^{-5}, 19,846$ (45.1\%) sequences of the 43,990 unigenes were found to have significant matches in the database and were assigned to 126 KEGG pathways. For some

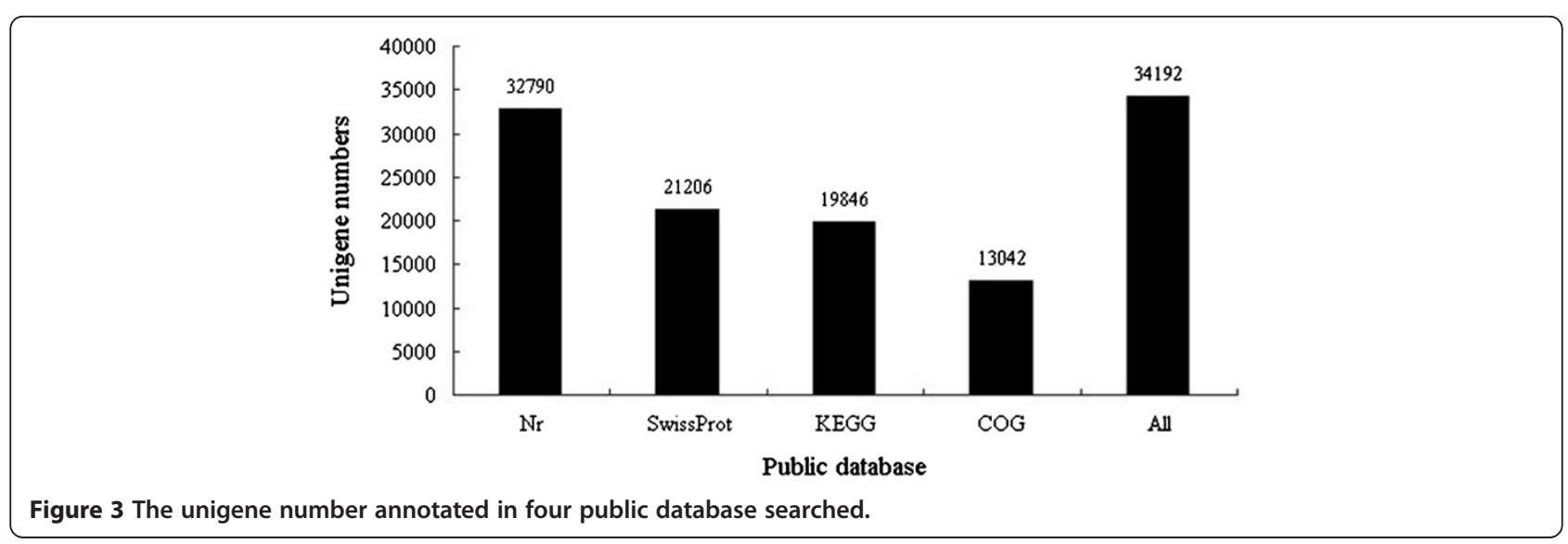




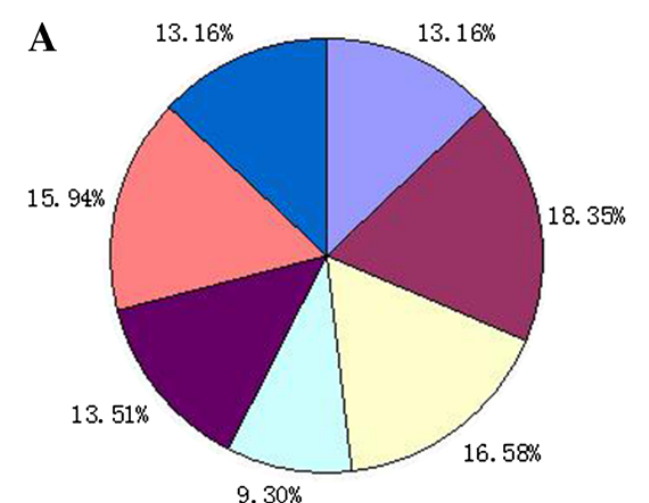

$$
\begin{aligned}
& \square 0 \\
& \square 0 \sim 1 e-100 \\
& \square 1 e-100 \sim 1 e-60 \\
& \square 1 e-60 \sim 1 e-45 \\
& \square 1 e-45 \sim 1 e-30 \\
& \square 1 e-30 \sim 1 e-15 \\
& \square 1 e-15 \sim 1 e-5
\end{aligned}
$$

B

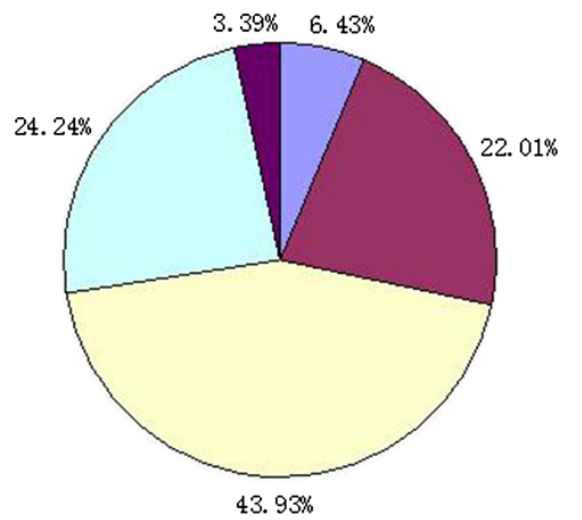

$$
\begin{aligned}
& \square 18 \% \sim 40 \% \\
& \square 40 \% \sim 60 \% \\
& \square 60 \% \sim 80 \% \\
& \square 80 \% \sim 95 \% \\
& \square 95 \% \sim 100 \%
\end{aligned}
$$

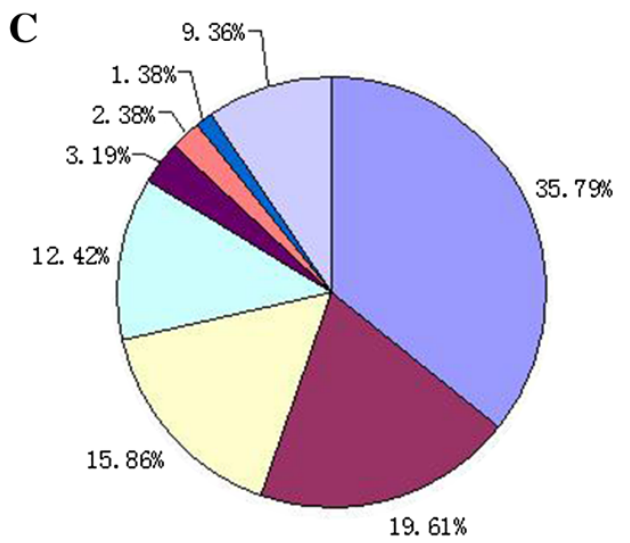

$\square$ Vitis virifera

$\square$ Ricimus commuris

$\square$ Populus trichocarpa

$\square$ Glycine max

- Medicago truncatula

$\square$ Arabidopsis thahiana

$\square$ Arabidopsis lyrata subsp. lyrata

$\square$ other

19. $61 \%$

Figure 4 Characteristics of similarity search of unigenes against Nr databases. (A) E-value distribution of BLAST hits for each unigene with a cutoff E-value of 1.0E-5. (B) Similarity distribution of the top BLAST hits for each unigene. (C) Species distribution of the top BLAST hits for each unigenes in $\mathrm{Nr}$ dababase.

genes being assigned to several KEGG pathways, there were 21,603 sequence hit in 126 KEGG pathways together. Of the 126 KEGG pathways, there were 25 pathways with over 200 unigenes assigned. The pathway of RNA transport was assigned the most unigene (1,287, 5.96\%), followed by plant-pathogen interaction (1,147, 5.31\%), endocytosis (979, 4.53\%), glycerophospholipid metabolism (947, 4.38\%), mRNA surveillance pathway (942, 4.36\%), plant hormone signal transduction $(928,4.30 \%)$, ether lipid metabolism (799, 3.70\%), spliceosome(745, 3.45\%), starch and sucrose metabolism $(565,2.62 \%)$, protein processing in endoplasmic reticulum (493, 2.28\%), whereas only no more than 10 unigenes were assigned to $\mathrm{C} 5$-branched dibasic acid metabolism, biotin metabolism, caffeine metabolism and betalain biosynthesis (Additional file 1: Table S1).

\section{Identification of the genes encoding cellulose synthase (CesA genes)}

As an important natural fiber, ramie fiber development is a major research area with a focus on improving the 


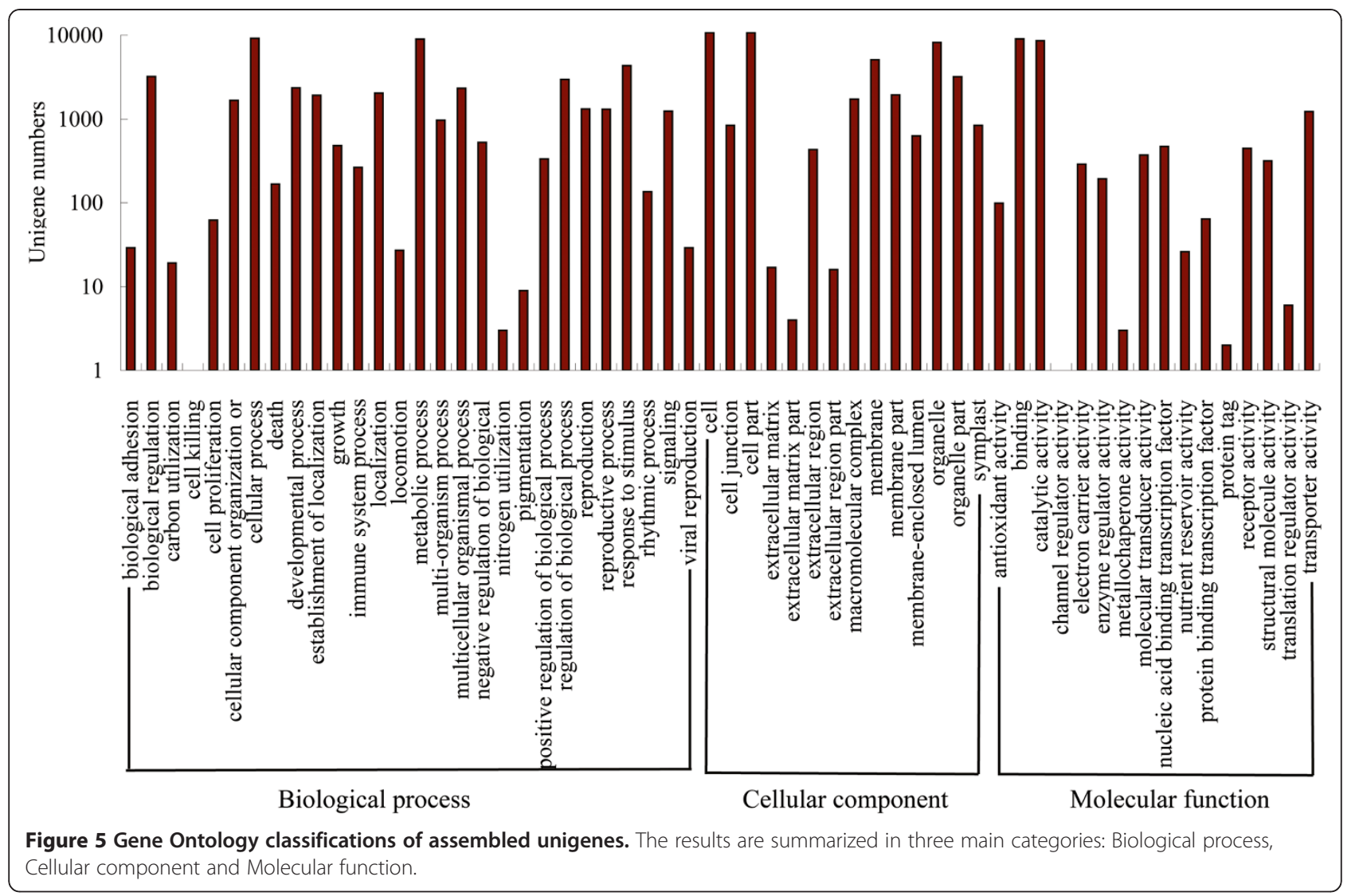

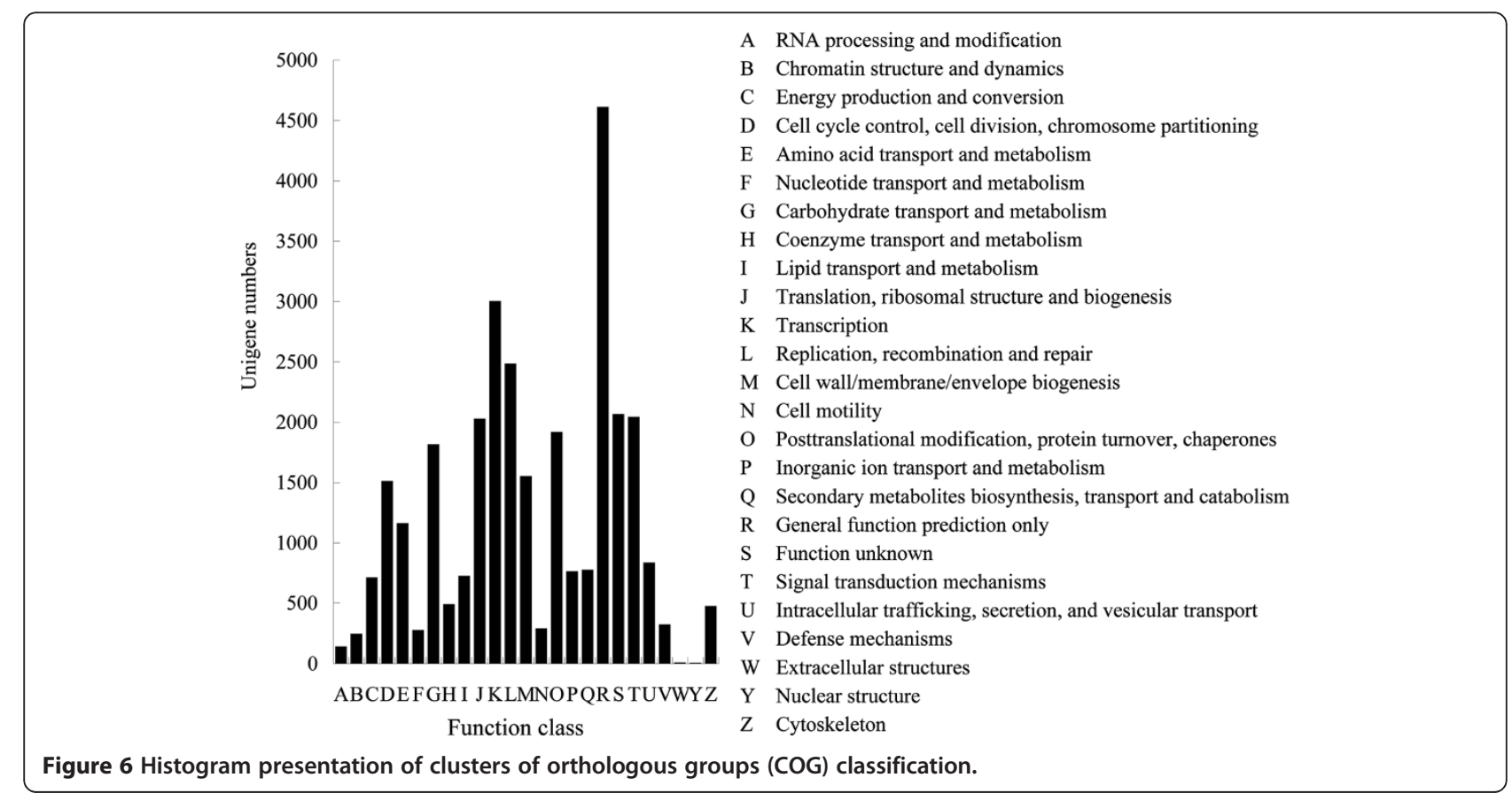


fiber yield and quality. Cellulose is a major component of the ramie fiber, and its content in the fiber significantly influences the yield and quality of ramie fiber. Out of 43,990 genes de novo assembled, 34,192 unigenes were annotated their function based on the sequence similarity search against the public databases. By searching the annotation with the keyword "cellulose synthase" from these 34,192 genes, 51 CesA genes were identified. Among these 51 genes, 30 unigenes have a significant homology (less than 1.0E-50) with the CesA gene of other species; 22 genes encoding proteins have their orthologous protein with more than $80 \%$ sequence similarity; 21 ramie CesA genes are homologous with the CesA gene of Vitis vinifera (Additional file 2: Table S2). Additionally, the conserved domain of ramie 51 CesA genes encoding protein was searched from the conserved domain database (CCD) and the result showed that 23 CesA gene-encoded proteins possessed the conserved domain of cellulose synthase. Interestingly, there are 12 genes assigned into the KEGG pathway of starch and sucrose metabolism involved in cellulose biosynthesis, and 39 genes were not assigned to any pathway (Additional file 2: Table S2).

In order to identify the potential candidates of the CesA genes involved in bast fiber biosynthesis, the expression levels of all 51 CesA genes in the stem bark, stem xylem, stem shoot and leaves were analyzed by RT-qPCR assay. The result showed that there were 36 CesA genes with relatively high expression levels in the stem bark from which the fiber was extracted (Additional file 2: Table S2). Among the 36 genes expressed in the bark, 33 genes showed higher expression levels in the bark than in other tissues (Figure 7); the Unigene14589 displayed a relatively high expression level in the stem bark and xylem and CL1547.contig1 showed similarly high expression levels in the stem bark, shoot and leaves, respectively (Figure 7); while the Unigene 21178 showed a constitutive expression in stem bark, shoot, xylem and leaf (Additional file 2: Table S2).

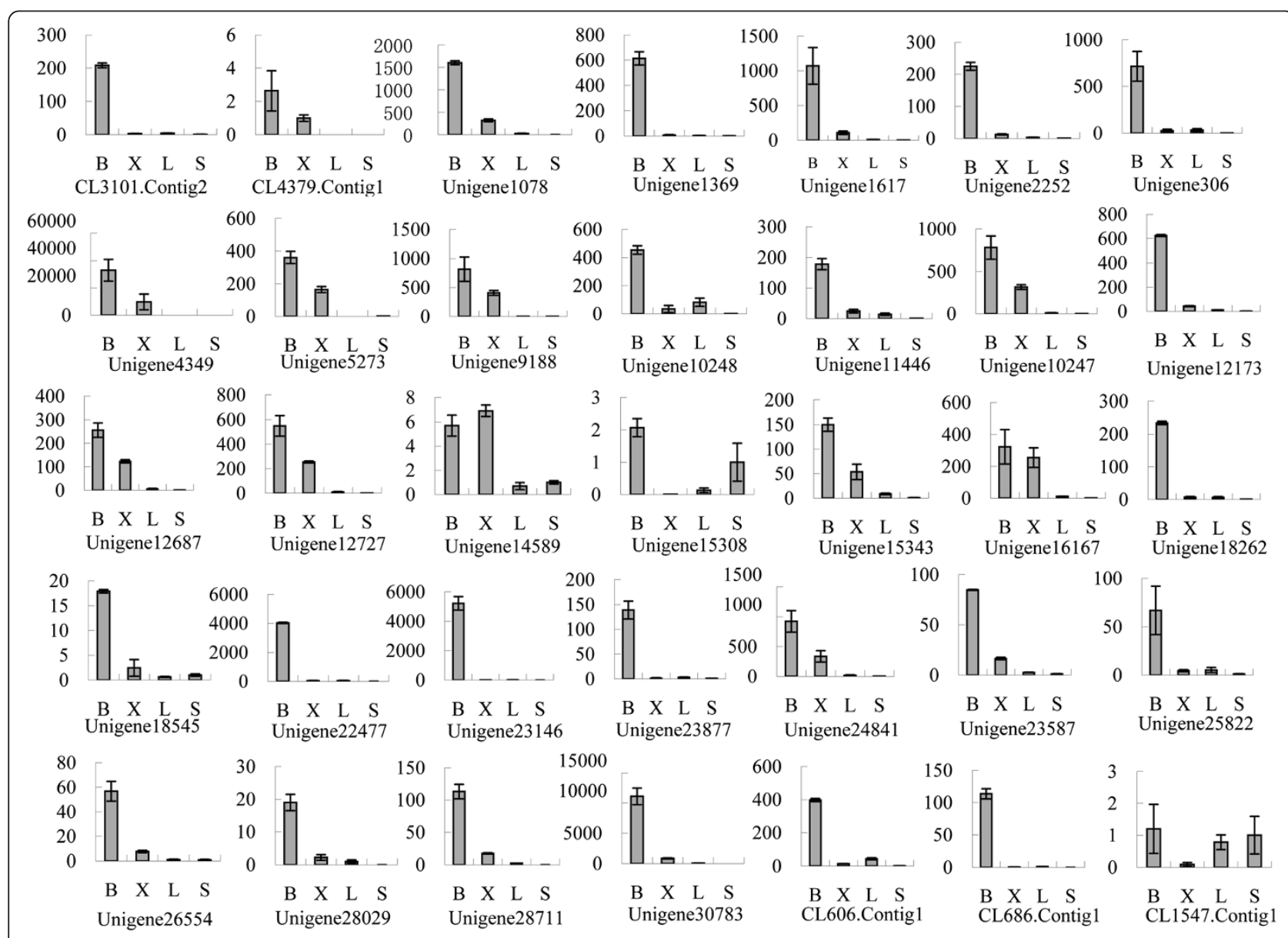

Figure 7 The expression pattern of CesA genes expressed with relative high level in stem bark. The $B, X, L$ and $S$ of $X$ axis represent the stem bark, stem xylem, shoot and leaf, respectively. 


\section{Discussion}

\section{Characterization of the ramie transcriptome and the discovery of 43,990 new genes}

With the development of sequencing technology, many plants such as Arabidopsis, rice, maize and sorghum have had their complete genome sequence [20-23], which has accelerated the research on functional genomics and accumulated the understanding of mechanisms that underlie plant growth and development. However, for some non-model plants and minor crops, it is infeasible to execute whole genome sequencing because of the expensive cost. The Next Generation Sequencing (NGS) provides an opportunity to mine the genes and deepen our understanding of growth and development in nonmodel plants. Recently, transcriptomes of scores of nonmodel species have been characterized [9-13], which expanded our understanding of gene expression, regulation and networks of important traits of the corresponding plants. Previously, only 418 ramie ESTs had been identified in database despite it being an important natural fiber crop. The lack on ramie gene severely hindered our understanding of ramie growth and fiber development, which presented an obstacle to improve the fiber yield and quality. In our study, the ramie transcriptome was characterized and in all 43,990 genes were newly discovered in ramie. Sequence orientation of approximately $78 \%$ of the unigenes discovered in this study was ascertained, along with the annotation of their function by searching against public databases. In addition, the functions of the unigenes were classified by COG and GO and the metabolic pathways were ascertained by using the KEGG database. Because of this study, the number of genes identified in ramie achieved a major leap from 418 to 43,990 . These results will immensely help us to explore the major genes for important agronomic traits in ramie, and further understand their regulation mechanisms, especially bastfiber formation and development mechanism.

In our study, the transcriptome sequencing was completed by using the Illumina Genome Analyser system platform HiSeq2000. HiSeq2000 can provide the sequence with a read-length of $90 \mathrm{bp}$, which is longer than that provided by other sequencing platform, such as GAII. In addition, the raw reads were stringently filtered before the de novo assembly. Theoretically, the reads with adaptor contamination can still be used after trimming the adaptor sequence. However, considering that the distance of two reads of paired-end was utilized to assemble the sequence by the software, there is an inaccurate distance between two paired-end reads with a length smaller than $90 \mathrm{bp}$, which may lead to a wrong assembly. In order to attain the sequences with high quality, all reads with adaptor contamination were discarded. Therefore, our transcriptome sequence has better quality than that of other species reported by previous studies [9-13], which was evident in several aspects. First, the average length of the unigenes was 824 bp which was far longer than that of other transcriptome sequences. Second, approximately $78 \%$ of the genes discovered in this study were successfully annotated for their functions. The ratio of genes annotated function was higher than that found in previous studies. Furthermore, we arrived at a higher ratio of genes assigned to the corresponding KEGG pathways. It was obvious that the transcriptome sequence generated in this study will be valuable for further ramie research.

Sequence similarities presented new clues for determining the phylogenetic relationship among ramie, Vitis vinifera, Glycine max, Ricinus communis and Populus trichocarpa.

Significant sequence similarities of $35.79 \%, 19.61 \%$, $15.86 \%$ and $12.42 \%$ between ramie and Vitis vinifera, Ricinus communis, Populus trichocarpa and Glycine max, respectively, were observed in present study. Ricinus communis and Populus trichocarpa belong to Malpighiales. Glycine max belongs to the order of Fabales. Ramie is classified into Urticaceae of Rosales. In plant taxonomy, Malpighiales, Fabales, Rosales are commonly placed in the superorder of rosids [24-26]. The close relationship among Malpighiales, Fabales and Rosales was further confirmed based on the evidence of sequence similarity evidence among the corresponding species of the three orders. However, the order of Vitaceae, including the species Vitis vinifera, had an unclear taxonomic position in the plant phylogenetic system. In the obsolete Angiosperm Phylogeny Group I (APG I) system, the Vitaceae was not classified [24]. In the revised yet no longer used APG II system, the Vitaceae was also unplaced to order, but only included in the superorder of rosids [25]. In the revised and updated APG III system, the rosids was classified as three subgroups: Vitales, fabids and malvids [26]. Malpighiales, Fabales and Rosales commonly belong to the fabids subgroup and Vitaceae belongs to Vitales. In other words, the relationship among Malpighiales, Fabales and Rosales is considered closer than that between Vitales and Rosales. However, in this study, new evidence at the molecular level has provided a contradictory taxonomic result. The ratio of transcriptome sequence similarity between ramie and Vitis vinifera was one-fold higher than that between ramie and the other three species, which suggests that the relationship between Vitales and Rosales is likely closer than that between Rosales, Malpighiales and Fabales.

\section{Potential candidate CesA genes involved in bast fiber biosynthesis}

The cellulose is a chain of glucose residues, and is the principal component of the plant cell walls. Biosynthesis of cellulose can be dissected as three steps: initiation of the sugar chain, elongation, and termination of the sugar 
chain [27]. The initiation and elongation cellulose is performed by cellulose synthase which is a rosette-shaped enzyme complex in the plant cell plasma membrane [28]. The catalytic subunits of cellulose synthase (CesAs) are central catalysts involved in the generation of plant cell wall cellulose [29,30]. CesA genes have been extensively studied in plants such as Arabidopsis, rice, cotton and barley [31-34]. Ramie bast fiber has highly enriched cellulose. However, only one ramie CesA gene has been identified based on the RACE technology [35]. In this study, with the help of transcriptome analysis and gene functional annotation, a total of 51 CesA genes were identified. Twenty-three CesA gene-encoded proteins have conserved domain of cellulose synthase. However, beacuse many EST sequences assembled in this study were not the full-length cDNA and protein sequences predicted according to these ESTs were incomplete, there are 28 CesA gene-encoded proteins that were not found their conserved domain. On the basis of the KEGG database searching, 12 CesA genes were assigned to the pathway of cellulose biosynthesis. Moreover, analysis of the expression pattern of 51 CesA genes showed that there are 36 genes with high expression levels in the stem bark. Of these 36 genes, 33 genes showed higher expression levels in the bark than in other tissues. It is likely that these 36 CesA genes are responsible for the biosynthesis of ramie fiber. Therefore, the identification of these genes will be helpful in further understanding of the mechanisms of ramie fiber development.

\section{Conclusion}

In this study, the ramie transcriptome was first characterized by de novo sequencing without the presence of a reference genome using Illumina paired-end sequencing technology. In all 43,990 genes with excellent sequence qualities were identified, which achieved a great leap in the knowledge of expressed sequence information of ramie. The substantial amount of transcripts obtained will certainly accelerate the understanding of the ramie growth and development mechanism, along with providing a strong basis for future genomic research. Furthermore, 51 CesA genes were mined in our research and their expression patterns were analyzed. Thirty-six CesA genes displayed relatively high expression levels in the stem bark, 33 of which showed higher expression in the bark than in other tissues. Identification of the CesA gene expressed in the stem bark will present an opportunity to understand the formation and development of ramie bast fiber, which provide a foundation for ramie breeding to improve the fiber yield and quality.

\section{Methods}

\section{Plant material and RNA extraction}

The elite ramie cultivar "Zhongzhu 1" was grown in the experimental field of the Institute of Bast Fiber
Crops, Chinese Academy of Agricultural Sciences, Changsha, China in 2012. Samples of tissues including leaves, root, stem bast, stem xylem and stem shoot were collected from 10-day-old seedling, 30-day-old ramie which have a vigorous vegetative growth and 60day-old ramie whose fiber is about to ripeness, respectively. The sampled tissues were immediately frozen in liquid nitrogen and stored at $-80^{\circ}$ until use. Total RNAs were extracted from each tissue of ramie of three growth stages using TRIzol reagent (Transgene Company, Illkirch Graffenstaden Cedex, France) according to the manufacturer's protocol. A total of $20 \mu \mathrm{g}$ of RNA was equally pooled from the five tissues for cDNA library preparation. The residual RNA was used for RT-qPCR.

\section{cDNA library construction and sequencing}

Illumina sequencing was performed at Beijing Genomics Institute (BGI)-Shenzhen, Shenzhen, China (http://www. genomics.cn/index.php) by using the HiSeq ${ }^{\text {TM }} 2000$ platform according to the manufacturer's instructions (Illumina, San Diego, CA). Briefly, poly (A) RNA was isolated from $20 \mu \mathrm{g}$ of total RNA using Sera-mag Magnetic Oligo (dT) Beads (Illumina). To avoid priming bias when synthesizing cDNA, the purified mRNA was first fragmented into small pieces. Then the double-stranded cDNA was synthesized using the SuperScript Double-Stranded cDNA Synthesis kit (Invitrogen, Camarillo, CA) with random hexamer (N6) primers (Illumina). The synthesized cDNA was subjected to end-repair and phosphorylation using T4 DNA polymerase, Klenow DNA polymerase and T4 PNK. These repaired cDNA fragments were 3' adenylated using Klenow Exo- (3' to 5' exo minus, Illumina). Illumina Paired-end adapters were ligated to the ends of these 3'-adenylated cDNA fragments. To select a size range of templates for downstream enrichment, the products of ligation reaction were purified on a $2 \%$ TAE-agarose gel (Certified Low-Range Ultra Agarose, Biorad). A range of cDNA fragments (200 $\pm 25 \mathrm{bp})$ was excised from the gel. Fifteen rounds of PCR amplification were performed to enrich the purified cDNA template using PCR Primer PE 1.0 and PE 2.0 (Illumina) with Phusion DNA Polymerase. The cDNA library was constructed with a fragment length range of $200 \mathrm{bp}( \pm 25 \mathrm{bp})$. Finally, after validating on an Agilent Technologies 2100 Bioanalyzer using the Agilent DNA 1000 chip kit, the cDNA library was sequenced on a PE flow cell using Illumina Genome Analyzer HiSeq 2000, and the workflow was as follows: template hybridization, isothermal amplification, linearization, blocking, sequencing primer hybridization, and sequencing on the sequencer for Read 1. After completion of the first read, the templates can be regenerated in situ to enable a second $90 \mathrm{bp}$ read from the opposite end of the fragments, i.e., the newly sequenced strands are stripped off and the complementary strands are bridge amplified to form clusters. Once the 
original templates are cleaved and removed, the reverse strands undergo sequencing-by-synthesis. The sequencing data are deposited in NCBI Sequence Read Archive (SRA, http://www.ncbi.nlm.nih.gov/Traces/sra) with accession number SRA057664.

\section{Data filtering and de novo assembly}

The quality requirement for de novo transcriptome sequencing is far higher than that for re-sequencing, because sequencing errors can create difficulties for the short-read assembly algorithm. We therefore carried out a stringent filtering process. Firstly, we removed reads that do not pass the built-in Illumina's software Failed-Chastity filter according to the relation "failed-chastity $\leq 1$ ", using a chastity threshold of 0.6 , on the first 25 cycles. Secondly, we discarded all reads with adaptor contamination. Thirdly, we ruled out low-quality reads with more than $5 \%$ ambiguous sequences "N". Finally, the reads with more than $20 \% \mathrm{Q}<20$ bases were also removed. De novo assembly was carried out using Trinity [14]. Additionally, if there are mutli-duplication's reads, only one read copy will be retained for assembly and redundant duplication reads be eliminated. Trinity assembled some reads which had overlapped nucleic acid sequence and generated contigs. To obtain the unigene, the paired-end reads were realigned to contigs, which can identify different contigs in the same transcript and ascertain the interval among these contigs. Then, these contigs in one transcript were assembled by the Trinity and gained the sequence not being extended on either end defined as unigenes. Then the TGICL [15] is used to get rid of redundant unigene and further assemble all the unigenes to form a single set of non-redundant unigenes.

Finally, BLASTx alignment (E value $<10^{-5}$ ) between unigenes and protein databases like NCBI non-redundant protein $(\mathrm{Nr})$ database http://www.ncbi.nlm.nih.gov, SwissProt protein database http://www.expasy.ch/sprot, the Kyoto Encyclopedia of Genes and Genomes (KEGG) pathway database http://www.genome.jp/kegg, and the Cluster of Orthologous Groups database http://www.ncbi.nlm.nih.gov/ COG was performed, and the best aligning results were used to decide the sequence direction of unigenes. If the results of different databases conflicted with each other, a priority order of Nr, Swiss-Prot, KEGG and COG should be followed when deciding the sequence direction of unigenes. When a unigene happened to be unaligned to none of the above databases, ESTScan software [36] was used to predict its coding regions as well as to decide its sequence direction.

\section{Gene annotation and analysis}

For annotation of unigenes using various bioinformatics approaches, the unigenes were firstly searched against the $\mathrm{Nr}$, COG, KEGG and Swiss-Prot protein database using local BLASTx with E value cutoff of $10^{-5}$. To estimate the number of annotated unigenes that matched to unique genes in the two databases, these files were filtered. With $\mathrm{Nr}$ annotation, Blast2GO program [18] was used to get $\mathrm{GO}$ annotation according to molecular function, biological process and cellular component ontologies http://www. geneontology.org. The sequences of the unigenes were also aligned to the COG database to predict and classify possible functions. Pathway assignments were carried out according to the Kyoto Encyclopedia of Genes and Genomes pathway database [37] also using BLASTx with E value threshold of $10^{-5}$. The conserved domain of CesA gene encoding protein was searched from the conserved domain database (CCD) in NCBI [38].

\section{RT-qPCR analysis of CesA genes expression pattern}

The RNA extracted for stem bark, xylem, shoot and leaf was used for RT-qPCR. For each sample, first-strand cDNAs were reverse-transcribed from RNAs treated with DNase I (Fermentas, Canada) using M-MuLV Reverse Transcriptase (Fermentas, Canada) according to the manufacturer's instructions. RT-qPCR was performed using an optical 96-well plate with an iQ5 multicolor real time PCR system (Bio-RAD, USA). Each reaction contained $1 \mu \mathrm{L}$ of cDNA template, $10 \mathrm{nM}$ gene-specific primers, $10 \mu \mathrm{L}$ of SYBR Premix Ex Taq, and 0.4 $\mu \mathrm{L}$ of ROX Reference Dye (FINNZYMES, Finland) in a final volume of $20 \mu \mathrm{L}$. The 18S rRNA gene was selected for the endogenous control [35]. The primer sequence of CesA genes and 18S rRNA gene were listed in Additional file 3: Table S3. The thermal cycle used was as follows: $95^{\circ} \mathrm{C}$ for $15 \mathrm{~min}$, followed by 40 cycles of $95^{\circ} \mathrm{C}$ for $10 \mathrm{~s}, 55^{\circ} \mathrm{C}$ for $20 \mathrm{~s}$ and $72^{\circ} \mathrm{C}$ for $30 \mathrm{~s}$. RT-qPCR was performed in triplicate for each sample. Relative expression levels were determined as described previously [39].

\section{Additional files}

Additional file 1: Table S1. Pathway assignment based on KEGG.

Additional file 2: Table S2. CesA gene identified in ramie.

Additional file 3: Table S3. Primer sequence of CesA and 18S RNA genes used for RT-qPCR.

\begin{abstract}
Abbreviations
GO: Gene ontology; RT-qPCR: Quantitative real-time polymerase chain reaction; CesA: cellulose synthase; KEGG: Kyoto Encyclopedia of Genes and Genomes Pathway database; EST: expressed sequence tag; NGS: next generation sequencing; Nr database: NCBI non-redundant protein database; COG: Cluster of Orthologous Groups; CCD: conserved domain database; BLAST: Basic Local Alignment Search Tool.
\end{abstract}

Competing interests

The authors have declared that no competing interests exist.

Authors' contributions

LT and TS conceived and designed the experiment. LT and ZS performed the experiment. TQ, CP and YY helped to prepare the reagents and materials. LT carried out the data analysis and wrote the manuscript. All authors read and approved the final manuscript. 


\section{Acknowledgements}

We acknowledge the Beijing Genomics Institute at Shenzhen for its assistance in original data processing and related bioinformatics analysis. This work was supported by grants from the National Natural Science Foundation of China (31101189), Natural Science Foundation of Hunan Province (10JJ3063), and National Modern Agro-industry Technology Research System (nycytx-19-E16).

Received: 2 September 2012 Accepted: 18 February 2013

Published: 26 February 2013

\section{References}

1. The Rice Full-Length cDNA Consortium: Collection, Mapping, and Annotation of Over 28,000 cDNA Clones from japonica Rice. Science 2003, 301:376-379.

2. Alexandrov N, Brover V, Freidin S, Troukhan M, Tatarinova T, Zhang H, Swaller T, Lu Y, Bouck J, Flavell R, Feldmann K: Insights into corn genes derived from large-scale cDNA sequencing. Plant Mol. Biol. 2009, 69:179-194

3. Soderlund C, Descour A, Kudrna D, Bomhoff M, Boyd L, Currie J, Angelova A, Collura K, Wissotski M, Ashley E, et al: Sequencing, Mapping, and Analysis of 27,455 Maize Full-Length cDNAs. PLoS Genetics 2009, 5:e1000740.

4. Zhang D, Choi D, Wanamaker S, Fenton R, Chin A, Malatrasi M, Turuspekov $Y$, Walia $H$, Akhunov E, Kianian $P$, et al: Construction and Evaluation of cDNA Libraries for Large-Scale Expressed Sequence Tag Sequencing in Wheat (Triticum aestivum L.). Genetics 2004, 168:595-608.

5. Meyer E, Aglyamova GV, Wang S, Buchanan-Carter J, Abrego D, Colbourne $\mathrm{JK}$, Willis BL, Matz MV: Sequencing and de novo analysis of a coral larval transcriptome using 454 GSFIx. BMC Genomics 2009, 10:219.

6. Wang J, Wang W, Li R, Li Y, Tian G, Goodman L, Fan W, Zhang J, Li J, Guo Y, et al: The diploid genome sequence of an Asian individual. Nature 2008, 456:60-65.

7. Huang YW, Huang TH, Wang LS: Profiling DNA methylomes from microarray to genome-scale sequencing. Technol. Cancer Res. Treat 2010, 9:139-147.

8. Nobuta K, McCormick K, Nakano M, Meyers BC: Bioinformatics analysis of small RNAs in plants using next generation sequencing technologies. Methods Mol. Biol. 2010, 592:89-106.

9. Barakat A, DiLoreto DS, Zhang Y, Smith C, Baier K, Powell WA, Wheeler N Sederoff R, Carlson JE: Comparison of the transcriptomes of American chestnut (Castanea dentata) and Chinese chestnut (Castanea mollissima) in response to the chestnut blight infection. BMC Plant Biol. 2009, 9:51.

10. Sun C, Li Y, Wu Q, Luo H, Sun Y, Song J, Lui EM, Chen S: De novo sequencing and analysis of the American ginseng root transcriptome using a GS FLX Titanium platform to discover putative genes involved in ginsenoside biosynthesis. BMC Genomics 2010, 11:262.

11. Wang Z, Fang B, Chen J, Zhang X, Luo Z, Huang L, Chen X, Li Y: De novo assembly and characterization of root transcriptome using Illumina paired-end sequencing and development of cSSR markers in sweetpotato (Ipomoea batatas). BMC Genomics 2010, 11:726.

12. Garg R, Patel R, Tyagi A, Jain M: De Novo Assembly of Chickpea Transcriptome Using Short Reads for Gene Discovery and Marker Identification. DNA Res. 2011, 18:53-63.

13. Deng Y, Yao J, Wang X, Guo H, Duan D: Transcriptome Sequencing and Comparative Analysis of Saccharina japonica (Laminariales, Phaeophyceae) under Blue Light Induction. PLoS ONE 2012, 7:e39704.

14. Grabherr M, Haas B, Yassour M, Levin J, Thompson D, Amit I, Adiconis X, Fan $L$, Raychowdhury $R$, Zeng $Q$, et al: Full-length transcriptome assembly from RNA-Seq data without a reference genome. Nature Biotechnology 2011, 29:644-652.

15. Pertea G, Huang X, Liang F, Antonescu V, Sultana R, Karamycheva S, Lee $Y$, White J, Cheung F, Parvizi B, et al: TIGR Gene Indices clustering tools (TGICL): a software system for fast clustering of large EST datasets. Bioinformatics 2003, 19:651-652

16. Altschul SF, Madden TL, Schaffer AA, Zhang J, Zhang Z, Miller W, Lipman DJ: Gapped BLAST and PSI-BLAST: a new generation of protein database search programs. Nucleic Acids Res. 1997, 25:3389-3402.

17. Cameron M, Williams HE, Cannane A: Improved gapped alignment in BLAST. IEEE/ACM Trans Comput. Biol. Bioinform 2004, 1:116-129.
18. Conesa A, Gotz S, Garcia-Gomez JM, Terol J, Talon M, Robles M: Blast2GO: a universal tool for annotation, visualization and analysis in functional genomics research. Bioinformatics 2005, 21:3674-3676.

19. Ye J, Fang L, Zheng H, Zhang Y, Chen J, Zhang Z, Wang J, Li S, Li R, Bolund L: WEGO: a web tool for plotting GO annotations. Nucleic Acids Res. 2006, 34:W293-297.

20. Initiative TAG: Analysis of the genome sequence of the flowering plant Arabidopsis thaliana. Nature 2000, 408:796-815.

21. Goff S, Ricke D, Lan T, Presting G, Wang R, Dunn M, Glazebrook J, Sessions A, Oeller P, Varma H, et al: A Draft Sequence of the Rice Genome (Oryza sativa L. ssp. japonica). Science 2002, 296:92-100.

22. Schnable P, Ware D, Fulton R, Stein J, Wei F, Pasternak S, Liang C, Zhang J, Fulton L, Graves T, et al: The B73 Maize Genome: Complexity, Diversity, and Dynamics. Science 2009, 326:1112-1115.

23. Paterson A, Bowers J, Bruggmann R, Dubchak I, Grimwood J, Gundlach H, Haberer G, Hellsten U, Mitros T, Poliakov A, et al: The Sorghum bicolor genome and the diversification of grasses. Nature 2009, 457:551-556.

24. Angiosperm Phylogeny Group (APG): An ordinal classification for the families of flowering plants. Ann. Mo. Bot. Gard 1998, 85:531-553.

25. Angiosperm Phylogeny Group (APG): An update of the Angiosperm Phylogeny Group classification for the orders and families of flowering plants: APG II. Bot. J. Linn. Soc 2003, 141:399-436.

26. Angiosperm Phylogeny Group (APG): An update of the Angiosperm Phylogeny Group classification for the orders and families of flowering plants. APG III. Bot J. Linn. Soc 2009, 161:105-121.

27. Read S, Bacic T: Prime Time for Cellulose. Science 2002, 295:59-60

28. Doblin M, Kurek I, Jacob-Wilk D, Delmer D: Cellulose Biosynthesis in Plants: from Genes to Rosettes. Plant Cell Physiol. 2002, 43:1407-1420.

29. Pear J, Kawagoe Y, Schreckengost W, Delmer D, Stalker D: Higher plants contain homologs of the bacterial celA genes encoding the catalytic subunit of cellulose synthase. Proc Natl Acad Sci USA 1996, 93:12637-12642.

30. Kumar M, Thammannagowda S, Bulone V, Chiang V, Han K, Joshi C, Mansfield S, Mellerowicz E, Sundberg B, Teeri T, et al: An update on the nomenclature for the cellulose synthase genes in Populus. Trends Plant Sci. 2009, 14:1360-1385.

31. Richmond T, Somerville C: The cellulose synthase superfamily. Plant Physiol. 2000, 124:495-498.

32. Kim H, Triplett B, Zhang H, Lee M, Hinchliffe D, Li P, Fang D: Cloning and characterization of homeologous cellulose synthase catalytic subunit 2 genes from allotetraploid cotton (Gossypium hirsutum L.). Gene 2012, 494:181-189.

33. Tanaka K, Murata K, Yamazaki M, Onosato K, Miyao A, Hirochika H: Three distinct rice cellulose synthase catalytic subunit genes required for cellulose synthesis in the secondary wall. Plant Physiol. 2003, 133:73-83.

34. Burton R, Shirley N, King B, Harvey A, Fincher G: The CesA gene family of barley quantitative analysis of transcripts reveals two groups of co-expressed genes. Plant Physiol. 2004, 134:224-236.

35. Tian Z, Yi R, Chen J, Guo Q, Zhang X: Cloning and Expression of Cellulose Synthase Gene in Ramie [Boehmerianivea (Linn.) Gaud.]. ACTA AGRONOMICA SINICA 2008, 34:76-83.

36. Iseli C, Jongeneel CV, Bucher P: ESTScan: a program for detecting, evaluating, and reconstructing potential coding regions in EST sequences. Intell. Syst. Mol. Biol: Proc. Int. Conf; 1999:138-148.

37. Kanehisa M, Goto S: KEGG: kyoto encyclopedia of genes and genomes. Nucleic Acids Res. 2000, 28:27-30.

38. Marchler-Bauer A, Lu S, Anderson J, Chitsaz F, Derbyshire M, DeWeese-Scott C, Fong J, Geer L, Geer R, Gonzales N: CDD: a Conserved Domain Database for the functional annotation of proteins. Nucleic Acids Res. 2011, 39:225-229.

39. Livak K, Schmittgen T: Analysis of relative gene expression data using real-time quantitative PCR and the 2(-Delta Delta $C(T)$ ) Method. Methods 2001, 25:402-408.

\section{doi:10.1186/1471-2164-14-125}

Cite this article as: Liu et al:: De novo assembly and characterization of transcriptome using Illumina paired-end sequencing and identification of CesA gene in ramie (Boehmeria nivea L. Gaud). BMC Genomics 2013 14:125. 